\title{
Envejecimiento cutáneo: causas y tratamiento
}

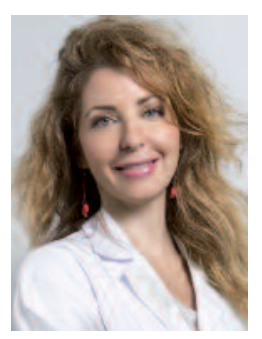

Elena González-Guerra

Doctora en Medicina.

Médico adjunto de Dermatología.

Hospital Clínico San Carlos. Madrid.

Profesora asociada de

Dermatología Médico-quirúrgica

y Venereología.

Departamento de Medicina.

Facultad de Medicina.

Universidad Complutense

de Madrid.

Profesora de Dermatología. Universidad Internacional de la Rioja (UNIR).

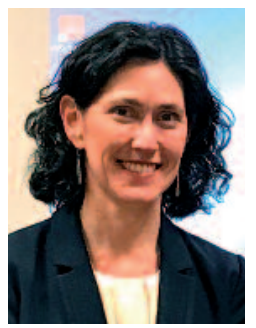

Tania Errasti Alcalá

Doctora en Medicina.

Colaborador clínico.

Departamento de Obstetricia

y Ginecología.

Clínica Universidad de Navarra.

Pamplona.

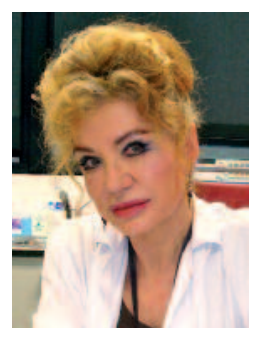

Aurora Guerra-Tapia

Doctora en Medicina.

Jefa de Sección de Dermatología.

Hospital Universitario

12 de Octubre. Madrid.

Profesora titular de Dermatología

Médico-quirúrgica y Venereología.

Universidad Complutense

de Madrid.

Profesora de Dermatología.

Universidad Internacional

de La Rioja (UNIR).

\section{RESUMEN}

El envejecimiento en general, y el cutáneo en particular, es un proceso deletéreo y universal, que, sin embargo, admite diferencias individuales en función de las causas que actúan sobre el proceso. No existe una causa única, admitiéndose diferentes teorías, entre las que se encuentran las teorías no estocásticas (no azarosas), como es la genética, en la que se incluye la de la senescencia replicativa y la de los gerontogenes; y las teorías estocásticas (azarosas), que hacen referencia al daño ambiental acumulativo a través de la oxidación y la glicación producida por tabaco, sol, contaminación y otros factores entre los que se encuentra el factor hormonal.

La prevención se fundamenta en una dieta sana, ejercicio físico y evitación de los factores ambientales como el tabaco y el sol mediante la fotoprotección. En el tratamiento, se consideran la terapia hormonal sustitutiva, los cosméticos y cosmecéuticos, las técnicas intervencionistas que se practican en la consulta (mesoterapia, implantes o rellenos, toxina botulínica, peeling químico, láser e hilos tensores) y la cirugía estética.

Palabras clave: vejez, envejecimiento cutáneo, causas, teoría estocástica, teoría no estocástica, fotoprotección, hormonas, cosméticos, rejuvenecimiento, técnicas dermoestéticas.

\section{ABSTRACT}

Aging in general, and cutaneous in particular, is a deleterious and universal process, which nevertheless admits individual differences depending on the causes that act on the process. There is no single cause, with different theories being accepted, such as non-stochastic (non-random) theories such as genetics, including replicative senescence and gerontogenes, and stochastic (random) theories that refer to cumulative environmental damage through oxidation and glycation produced by tobacco, sun, pollution and other factors including hormone factor.

Prevention is based on a healthy diet, physical exercise and avoidance of environmental factors such as tobacco 
and the sun by photoprotection. The treatment includes hormone replacement therapy, cosmetics and cosmeceuticals, interventional techniques to be practiced in consultation (mesotherapy, implants or fillers, botulinum toxin, chemical peeling, laser and tensioning threads), and cosmetic surgery.

Keywords: aging, cutaneous aging, causes, stochastic theory, non-stochastic theory, photoprotection, hormones, cosmetics, rejuvenation, dermaesthetic techniques.

\section{VEJEZ: CONCEPTO}

Según el diccionario de la Real Academia Nacional de Medicina $^{1}$, la vejez — edad avanzada, edad senil, tercera edad, ancianidad, senectud, senilidades el «período final de la vida humana, caracterizado por la progresiva declinación de las funciones fisiológicas en tejidos, órganos, aparatos y sistemas, con cambios cognitivos, afectivos y de personalidad, y pérdida de la capacidad de adaptación del individuo a los factores ambientales negativos que le rodean. Se considera vejez el período de la vida a partir de los 65 años, si bien determinados factores socioculturales y sanitarios, aparte de las condiciones genéticas, pueden retrasar el proceso y la edad de envejecimiento». Las características que la definen son:

1. Es un proceso deletéreo, esto es, perjudicial para la salud, que puede causar la muerte.

2. Es universal, puesto que afecta a todos los seres vivos.

3. Es heterogéneo, porque cada especie tiene un tiempo y una forma de envejecer. Existen patrones específicos de vejez para cada una de ellas.

4. Es individual, porque cada ser vivo dentro de cada especie tiene diferencias en su desarrollo evolutivo hacia el fin de la vida.

En esta revisión, nos dedicaremos solo a la especie humana.

\section{VEJEZ: CAUSAS}

El porqué de la vejez oculta todavía muchos misterios. Ante la pregunta "¿por qué un ratón vive dos años de media, y una tortuga, 200 años?», la respuesta primaria es: por causas genéticas. Pero no todo lo explica la genética si, por poner un ejemplo, observamos como la vida media de los perros de compañía se prolonga en función de la alimentación, el ejercicio y los estímulos mentales que reciba. Lo cierto es que no existe un consenso absoluto, pero sí una aproximación en las teorías que comentaremos a continuación.

\section{Teorías no estocásticas o deterministas}

Hacen referencia a aquellas que no dependen del azar, de la casualidad, sino que están predeterminadas. Según estas teorías, el envejecimiento está predeterminado, como lo está la adolescencia o la madurez. Es la etapa final de la programación de la vida, contenida en el genoma.

\section{Límite de Hayflick}

En 1961, los investigadores Hayflick y Moorhead observaron en un cultivo de fibroblastos (células del tejido conjuntivo especializadas en la síntesis de fibras colágenas y de glucoproteínas) que eran capaces de llevar a cabo un número finito de duplicaciones. Llegado este momento, las células perdían su potencial replicativo y entraban en una fase de «senescencia replicativa». Se enunció de este modo el límite de Hayflick como el número de duplicaciones que puede sufrir una célula eucariota antes de entrar en senescencia ${ }^{2}$. Se excluyen de esta norma los gametos sexuales.

\section{Senescencia replicativa}

Si las células tienen un límite de replicación, aparece la nueva pregunta: ¿cómo ocurre?, ¿de quién es la culpa? 
En este punto, hay que considerar el papel de los telómeros, que son los extremos de cada uno de los brazos del cromosoma, responsables del mantenimiento de la integridad cromosómica ${ }^{3}$. Con cada división celular sucesiva, los telómeros se acortan, y llega un momento en que el cromosoma no puede mantenerse íntegro, y la célula deja de dividirse, entrando en senescencia. Las células embrionarias, las células madre y algunas células cancerosas mantienen la longitud de los telómeros y, por lo tanto, la inmortalidad celular.

\section{Gerontogenes}

A lo largo de la vida, muchas células se dañan y sufren errores genéticos (mutaciones). Algunas de ellas mueren por un fenómeno de apoptosis. Pero otras son reparadas por los genes reparadores. Los genes implicados en la reparación de daños se alteran con el paso del tiempo, creándose una incapacidad para reparar los errores genéticos, que se acentúan con la edad del individuo $^{4}$.

\section{En la piel}

También en la piel existe expresión genética de datos que participan en el envejecimiento ${ }^{5}$. Así, mediante PCR (reacción en cadena de la polimerasa), se puede realizar un análisis del polimorfismo de los genes, encontrando, por ejemplo, genotipos de riesgo hacia más metaloproteinasas, que favorecen una mayor degradación de colágeno, o hacia menos acuaporinas, que llevan a menor hidratación, etcétera.

\section{Teorías estocásticas o del azar}

Hacen referencia al azar o casualidad, que va produciendo una serie de fenómenos acumulativos y perjudiciales.

\section{Radicales libres}

Un radical libre es un fragmento de una molécula que posee un electrón sin aparear, muy reactivo, que participa como intermediario en las reac- ciones químicas e interviene en multitud de procesos biológicos. Se generan en el organismo como resultado de procesos metabólicos o agresiones externas y producen daño celular y genético.

Los principales agentes causales de radicales libres y acción en la piel son la radiación ultravioleta, el tabaco, las radiaciones ionizantes, la contaminación atmosférica, todos los procesos endógenos metabólicos fisiológicos y los que cursen con inflamación.

Con los radicales libres, se produce un daño ambiental acumulativo a través de la oxidación ${ }^{6}$, con la generación de ERO (especies reactivas de oxígeno), y de la glicación ${ }^{7}$, con la generación de AGE (advanced glycation end-products, productos de la glicación avanzada, esto es, reacción entre un azúcar reductor y los tejidos). Ocasionan un daño importante en las sirtuínas (enzimas reparadoras del ácido desoxirribonucleico presentes en todas las células) y en los telómeros, favoreciendo el envejecimiento.

Frente a los radicales libres, existen numerosos mecanismos de defensa naturales, llamados antirradicales libres. Así, existen sistemas enzimáticos (superóxido-dismutasa, catalasa, glutatión-peroxidasa, sistema tiorredoxina) y sistemas no enzimáticos (glutatión, vitamina $\mathrm{E}$, vitamina $\mathrm{C}$, coenzima $\mathrm{Q}$, ácido úrico, carotenoides y vitamina $\mathrm{A}$, ácido lipoico, selenio, cinc, flavonoides, fitoestrógenos), entre otros.

\section{Acumulación de productos de desecho o del desgaste}

Se produce una acumulación a lo largo de la vida de productos de desecho, algunos de los cuales pueden ser perjudiciales para la vida ${ }^{6}$.

\section{En la piel}

El daño ambiental acumulativo productor de ERO y AGE aumenta la producción de metaloproteinasas, que rompen el colágeno; inducen el factor de transcripción kappa $\mathrm{B}$, que produce mediadores inflamatorios, y aumentan el ácido ribonucleico mensajero de la elastina en los fibro- 
blastos dérmicos, que producen cambios elastolíticos ${ }^{5}$.

\section{EL TABACO COMO FACTOR CAUSAL DE ENVEJECIMIENTO CUTÁNEO}

Los efectos del tabaco sobre la piel en el envejecimiento son múltiples ${ }^{8,9}$ :

- Pérdida de luminosidad y tono. La nicotina provoca vasoconstricción y disminución de la circulación periférica. El monóxido de carbono del humo, al ser 200 veces más afín por la hemoglobina que el oxígeno, se une a la hemoglobina desplazando al oxígeno, por lo que disminuye la cantidad de oxígeno que llega a los tejidos periféricos, como la piel.

- Arrugas. El tabaco colabora en la formación de arrugas de expresión por el movimiento facial repetitivo (fruncir los labios, achinar los ojos, subir la frente...) durante el gesto de fumar. Además, produce alteración de las fibras elásticas de la dermis, al romperse el equilibrio entre la elastasa y el inhibidor de la alfa 1-proteinasa, que lleva a un incremento de la liberación de la elastasa por los neutrófilos y los macrófagos, a la vez que a una inactivación por oxidación de su inhibidor ${ }^{10}$. Estos hechos se producen incluso en la piel no expuesta al humo del tabaco $^{11}$.

- Alteraciones de la cicatrización. Se producen un mayor número de necrosis y dehiscencias en las heridas quirúrgicas, siendo de uso común la recomendación de suprimir el tabaco como mínimo tres semanas antes y cinco semanas después de una intervención quirúrgica ${ }^{12}$.

\section{LA RADIACIÓN ULTRAVIOLETA COMO FACTOR CAUSAL DE ENVEJECIMIENTO CUTÁNEO}

Aunque el sol emite prácticamente todo el espectro electromagnético, desde un punto de vista clínico y fotobiológico, la radiación de importancia para la piel es la ultravioleta (UV) y, dentro de ella, la UVB (290-320 nm) y la UVA (320-400 nm), la luz visible $(400-800 \mathrm{~nm})$ y la infrarroja $(800-$ $1700 \mathrm{~nm}$ ). El resto de la radiación emitida, incluida la UVC es filtrada por la atmósfera y no alcanza la superficie terrestre ${ }^{13}$. La dosis de irradiación recibida en un determinado punto de la superficie terrestre y durante un determinado momento depende de múltiples factores: latitud, altura sobre el nivel del mar, época del año, presencia de nubes o partículas en suspensión, y el espesor de la capa de ozono. También hay que considerar el riesgo de la exposición solar indirecta, producida por la radiación emitida por superficies reflectantes como la nieve, la hierba, la arena o el agua del mar. Todos estos factores van a incidir sobre una piel con diferente capacidad de broncearse y con diferente susceptibilidad a sufrir quemaduras solares (fototipos cutáneos).

Los efectos en cuanto a envejecimiento cutáneo de la radiación solar son numerosos ${ }^{14}$ :

- Lentigos. Aparecen máculas lenticulares hiperpigmentadas en zonas expuestas (fig. 1).

- Arrugas. Son fundamentalmente arrugas finas y superficiales de nueva creación, así como empeoramiento de las arrugas de expresión, estáticas, de la gravedad y del sueño.

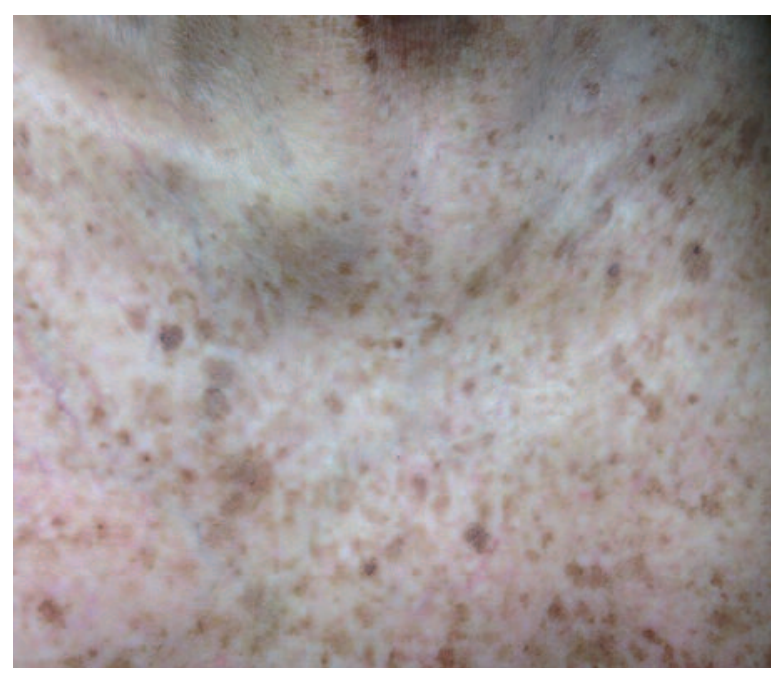

Figura 1. Lentigos como manifestación del envejecimiento cutáneo por la radiación ultravioleta. 
- Dermatoporosis. Consiste en un conjunto de datos como piel fina, traslúcida, con alteraciones de la pigmentación, pseudocicatrices estrelladas, y púrpura maculosa ${ }^{15}$ (fig. 2).

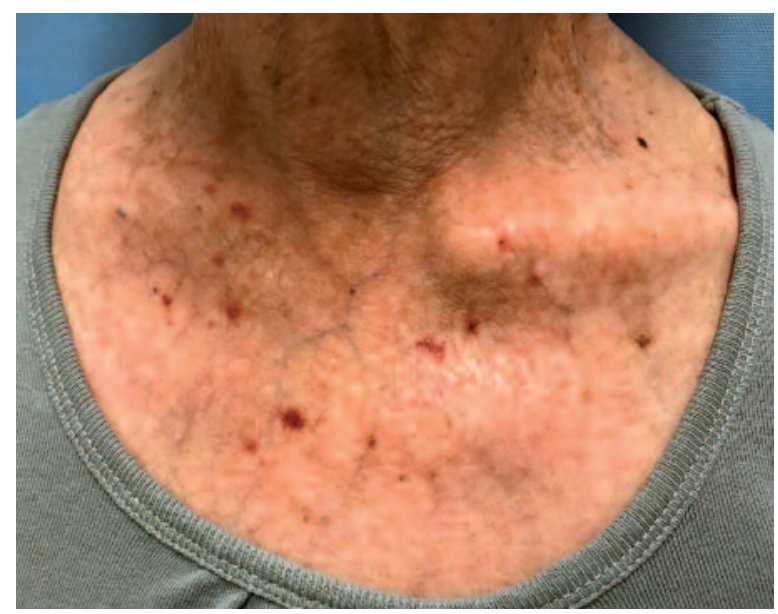

Figura 2. Dermatoporosis.

- Cáncer cutáneo. Es el grado máximo de envejecimiento cutáneo. La radiación UVB se relaciona directamente con la carcinogénesis. La franja de radiación UVA, tradicionalmente asociada al fotoenvejecimiento, de mayor longitud de onda y mayor penetración, es un carcinógeno importante para el compartimento de células madre de la piel.

\section{LOS CAMBIOS HORMONALES COMO FACTOR CAUSAL DE ENVEJECIMIENTO CUTÁNEO}

La piel es un órgano endocrino capaz de producir andrógenos, estrógenos a partir de los andrógenos gracias a la actividad de la aromatasa, y vitamina D gracias a la radiación UV sobre los queratinocitos.

También la piel es un órgano diana de múltiples hormonas que afectan en mayor o menor medida a su envejecimiento.

Es bien conocida la afectación de la piel en pacientes con acromegalia, por exceso de secre- ción de hormona de crecimiento (GH), y que el déficit aislado de $\mathrm{GH}$ produce signos de envejecimiento temprano como piel seca, delgada y arru$\operatorname{gada}^{16}$. Sin embargo, las hormonas que más afectan al envejecimiento de la piel son los esteroides sexuales: los andrógenos y los estrógenos.

\section{Andrógenos}

Hay receptores de andrógenos en queratinocitos, adipocitos, glándulas sudoríparas, fibroblastos y melanocitos. Los andrógenos modulan el grosor de la dermis y la epidermis, aumentan la producción grasa y el tamaño de las glándulas sebáceas. Son fundamentales en los cambios que se producen durante la pubertad, induciendo el desarrollo del vello terminal de los folículos pilosos de zonas androgénicas - pubis y axilas - y, en los varones, la barba, y favorecen el acortamiento del anágeno en el cuero cabelludo (alopecia). El déficit de andrógenos que se produce a lo largo de la vida de los varones y de las mujeres favorece la sequedad de la piel y las mucosas, y la disminución de los folículos pilosos del cuero cabelludo.

\section{Estrógenos}

En 1990, se identificaron receptores de estrógenos en queratinocitos epidérmicos, fibroblastos dérmicos, vasos sanguíneos y folículos pilosos. Actúan en los fibroblastos por el aumento de la transcripción de genes con dominios AP-1 relacionados con el área de unión del complejo Jun/Fos, y tienen poder antioxidante, reduciendo la producción de ERO, por lo que protegen la piel del envejecimiento intrínseco mediado por estos mecanismos.

Las hormonas esteroideas circulan unidas a globulinas transportadoras (globulina fijadora de hormonas sexuales [SHBG], hormona liberadora de corticotropina y albúmina) y la hormona activa es la fracción que circula libre. Existen circunstancias en las que se modifica la producción de estas proteínas transportadoras y entonces la concentración de las hormonas esteroideas se modifica. Durante la menopausia, en que la mujer deja de 
producir estrógenos, se reduce la concentración de SHBG, aumentando los andrógenos libres y favoreciendo sus efectos cutáneos, como son la aparición de vello terminal en zonas androgénicas de la cara en mujeres mayores, y sequedad de la piel.

Los cambios debidos al envejecimiento que se producen en la piel de la mujer tanto por la edad como los relacionados con el déficit de estrógenos se asocian más al tiempo transcurrido desde el cese de la producción de estrógenos que a la edad de la mujer, lo cual hace pensar que el envejecimiento intrínseco se debe más a la falta de estrógenos que a otros factores que también comparten los varones. Los cambios son tanto macroscópicos como microscópicos. Se ha demostrado la pérdida del 1,5\% de elasticidad cada año de déficit hormonal ${ }^{17}$, y del 1,1\% de grosor y el 2,1\% de colágeno también por año ${ }^{18}$.

\section{ENVEJECIMIENTO CUTÁNEO: PREVENCIÓN Y TRATAMIENTO}

La prevención del envejecimiento en general se sustenta en una dieta sana, ejercicio físico y evitar aquellos factores ambientales como el tabaco y el sol mediante la fotoprotección.

\section{Fotoprotección}

La fotoprotección es el conjunto de medidas que intentar eludir los efectos deletéreos de la radiación UV sobre la piel. La fotoprotección natural es la que recomienda evitar el sol entre las 11:00 y las 15:00 horas solares y llevar una vestimenta adecuada consistente en tejido de trama tupida, no elástico, preferiblemente de algodón, de color oscuro y acompañado de un sombrero con ala. Las gafas de sol para UV son un complemento que protege del daño ocular.

Además de esta, existe la fotoprotección farmacológica, representada por los filtros solares o fotoprotectores, que cumplen una doble misión: preventiva del fotoenvejecimiento y la fotocarcinogénesis, y terapéutica en los casos de fotosensibilidad patológica.
Los fotoprotectores tópicos ${ }^{19}$ son preparaciones para aplicación externa que contienen sustancias (orgánicas o inorgánicas) que detienen parcialmente las radiaciones UV. Se preparan en una base líquida (soluciones, geles), semilíquida (cremas) o sólidas (barras o stick). Las cualidades que debe poseer un fotoprotector para conseguir la máxima eficacia son:

- Potencia fotoprotectora, representada por el factor de protección solar (índice de protección, factor de protección o FPS).

- Resistencia al agua.

- Sustantividad. Es la capacidad para mantener una protección prolongada en condiciones normales de utilización. Un fotoprotector tiene una buena sustantividad cuando se mantiene adherido a la piel pese a la sudación y la actividad física.

- Fotoestabilidad. Es la resistencia a la degradación por la luz. Un fotoprotector debe ser estable frente a la luz UV.

- Cosmética. Debe tener características de tacto, brillo, color y facilidad de aplicación que hagan agradable su uso.

\section{Hormonas}

La prevención y tratamiento hormonal del envejecimiento cutáneo con mayor evidencia científica es la terapia hormonal sustitutiva (THS).

Los ensayos publicados más antiguos (entre 1994 y 2007) sobre los efectos del tratamiento hormonal para prevenir el envejecimiento ofrecen resultados diversos, aunque no son ensayos aleatorizados, prospectivos y controlados con placebo, por lo que, junto con el bajo número de pacientes de algunos y el corto tiempo de seguimiento de otros, es difícil llegar a una conclusión.

En relación con el envejecimiento microscópico, Callens et al..$^{20}$ trataron a 98 mujeres con estradiol en gel o parches $(1,5 \mu \mathrm{g} /$ día), con un seguimiento de 4,8 años, y demostraron un aumento en el grosor de la piel del 7-15\% medido por ecografía. Por otra parte, Haapasaari et al. ${ }^{21}$ compa- 
raron en un estudio paralelo no aleatorizado a 18 mujeres con THS con estradiol y acetato de noretisterona o solo estradiol con o sin tratamiento, todas con menopausia reciente durante 12 meses, y no observaron cambios en la cantidad de colágeno ni proporción de fibras elásticas en la biopsia realizada en la piel del abdomen. Más recientemente, Sauerbronn et al. ${ }^{22}$, en un estudio aleatorizado, doble ciego, controlado con placebo, compararon a 41 mujeres tratadas con estradiol y acetato de ciproterona frente al placebo durante seis meses, e hicieron una evaluación histológica de la piel del brazo, encontrando diferencias estadísticamente significativas entre el contenido de colágeno (aumento del 6,49\%) y elastina, la relación área de epidermis/longitud de la membrana basal, y la relación queratinocitos/longitud de la membrana basal entre los dos grupos. Entre los restantes estudios, el de Owen et al. ${ }^{23}$ es un estudio aleatorizado, doble ciego, controlado con placebo, subsidiario del estudio KEEPS (Kronos Early Estrogen Prevention Study) para la prevención de enfermedades cardiovasculares, en el que se evaluaba a mujeres con distintos tipos de tratamiento (estrógenos conjugados equinos, estradiol transdérmico) frente a mujeres sin tratamiento, no encontrándose diferencias significativas (salvo la comparación por raza). Extraer una conclusión puede resultar difícil y, antes de aconsejar el tratamiento como medida preventiva, convendría disponer de más estudios aleatorizados, doble ciegos, controlados, con un número mayor de participantes.

En resumen, se ha demostrado que la THS sistémica con estrógenos locales o sistémicos (añadiendo o no gestágenos) induce cambios microscópicos, protegiendo del envejecimiento de la piel. Sin embargo, no se ha demostrado que la THS frene el envejecimiento macroscópico.

\section{Cosméticos y cosmecéuticos}

Aunque son muchos los productos cosméticos y cosmecéuticos invocados $^{15,24,25}$, señalaremos los siguientes con mayor evidencia científica:
- Retinoides, que actúan a través de la unión a los receptores de ácido retinoico (RAR) y/o a los receptores X de retinoide ( $\mathrm{RXR})$, implicados en la formación de colágeno de tipo I y III y en la inhibición de las metaloproteinasas de la matriz, que llevan al fotoenvejecimiento.

- Polifenoles, existentes en los extractos de plantas, que ejercen su acción antienvejecimiento a través de la neutralización de radicales libres, inhibición de la ciclooxigenasa y lipooxigenasa.

- Péptidos, que son pequeñas secuencias de aminoácidos. Son de señal (activan las señales celulares que desencadenan la síntesis de colágeno por parte de los fibroblastos), transportadores (estabilizan y transportan el cobre, un elemento importante para múltiples reacciones enzimáticas, la angiogénesis y la curación de heridas) y bloqueantes de neurotransmisores (pretenden bloquear la liberación de acetilcolina a nivel de la unión neuromuscular a imitación de la toxina botulínica).

- Vitaminas. Entre ellas, la vitamina C o ácido ascórbico, factor esencial para las dos enzimas que se requieren en la síntesis de colágeno: la propil hidroxilasa (estabiliza la molécula) y la lisil hidroxilasa (fortalece su estructura formando enlaces cruzados). Además, estimula la síntesis de procolágeno de tipo I en cultivos de fibroblastos humanos. También parece influir en la síntesis de elastina, disminuyendo su fabricación por los fibroblastos.

- Alfa-hidroxiácidos. Se consideran estimulantes del colágeno a concentraciones inferiores al $25 \%$. Entre el 50 y el $70 \%$, producen despegamiento de la epidermis, por lo que se utilizan como exfoliantes epidérmicos.

\section{Técnicas dermoestéticas practicadas en la consulta}

Además de las arrugas como principal manifestación del envejecimiento cutáneo, con las diversas técnicas, se intenta corregir de manera drástica otros cambios cutáneos relacionados con el envejecimiento como son la disminución y el descen- 


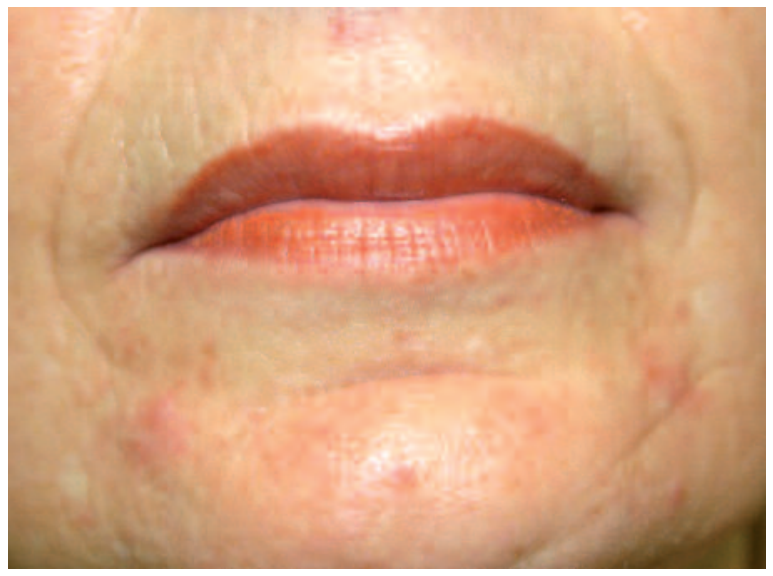

Figura 3. Envejecimiento cutáneo: arrugas de expresión en surcos nasogenianos y zona periorbicular.

so de las almohadillas de grasa malar, la alteración del contorno de los pómulos, la depresión de las mejillas, la acentuación del surco de la ojera, del surco nasolabial y la comisura perioral, así como la pérdida del contorno del óvalo facial. Todas ellas se pueden aplicar en consulta dermatológica, de forma ambulatoria, con escasos efectos secundarios.

Entre ellas, se encuentran ${ }^{26}$ :

- Mesoterapia, mediante inyecciones intradérmicas de sustancias activas sobre la piel (ácido hialurónico, vitaminas, minerales), que pretende mejorar la calidad de la piel con mayor eficacia que los cosméticos o cosmecéuticos.

- Implantes o rellenos, preferiblemente, reabsorbibles (ácido hialurónico, colágeno, hidroxiapatita cálcica, ácido poliláctico, etc.), que modifican los volúmenes alterados de forma no permanente (figs. 3 y 4 ).

- Toxina botulínica, que actúa produciendo la quimiodenervación temporal y pérdida de la actividad del órgano diana durante varios meses, con la finalidad de suavizar la expresión, modificar gestos antiestéticos y minimizar arrugas de expresión.

- Peeling químico (quimioabrasión, quimioexfoliación) con sustancias exfoliantes, pretendien-

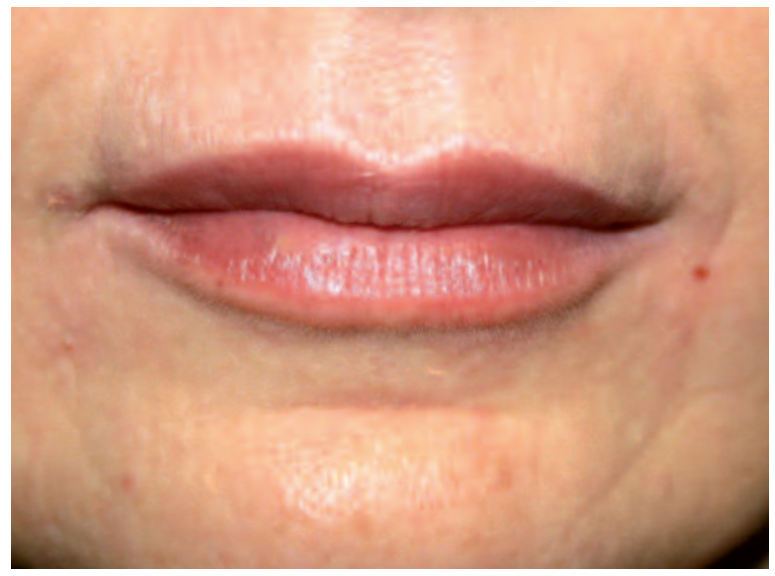

Figura 4. Resultado inmediato tras la infiltración con ácido hialurónico de las arrugas de la paciente de la figura 3.

do una renovación cutánea como tratamiento de hiperpigmentaciones o arrugas.

- Láser, que, dependiendo del tipo y uso, produce mejora de las arrugas superficiales, de las hiperpigmentaciones y de la calidad de la piel.

- Hilos tensores, que, introducidos en la dermis, tensan y remodelan los contornos faciales y corporales. Son reabsorbibles en unos seis meses, consiguiendo creación de nuevo colágeno de forma muy efectiva ${ }^{27}$.

\section{Cirugía estética}

Es una técnica de rejuvenecimiento sumamente eficaz, que debe ser practicada por un especialista en cirugía plástica, estética y reparadora.

Es objeto de otro artículo en este mismo número de la revista Más Dermatología.

\section{BIBLIOGRAFÍA}

1. Real Academia Nacional de Medicina. Diccionario de términos médicos. [Internet]. Madrid: Editorial Médica Panamericana; 2012. Disponible en: http://dtme.ranm.es/buscador.aspx?NIVEL_BUS=3\&LEMA_BUS=vejez

2. Luft FC. Approaching the Hayflick limit. Trends Cardiovasc Med. 2015;25(3):240-2

3. Rousseau P, Autexier C. Telomere biology: Rationale for diagnostics and therapeutics in cancer. RNA Biol. 2015;12(10): 1078-82.

4. Nyström T, Yang J, Molin M. Peroxiredoxins, gerontogenes linking aging to genome instability and cancer. Genes Dev. 2012;26(18):2001-8. 
5. Li T, Yan X, Jiang M, Xiang L. The comparison of microRNA profile of the dermis between the young and elderly. J Dermatol Sci. 2016;82(2):75-83.

6. Kammeyer A, Luiten RM. Oxidation events and skin aging. Ageing Res Rev. 2015;21:16-29.

7. Yokota M, Tokudome Y. The effect of glycation on epidermal lipid content, its metabolism and change in barrier function. Skin Pharmacol Physiol. 2016;29(5):231-42.

8. Freiman A, Bird G, Metelitsa Al, Barankin B, Lauzon G). Cutaneous effects of smoking. J Cutan Med Surg. 2004;8(6): 415-23.

9. Just-Sarobé M. Smoking and the skin. Actas Dermosifiliogr. 2008;99(3):173-84.

10. Francès $C$, Boisnic $S$, Hartmann DJ, Dautzenberg B, Branchet $M C$, Le Charpentier YL, et al. Changes in the elastic tissue of the non-sun-exposed skin of cigarette smokers. Br J Dermatol. 1991;125(1):43-7.

11. Just $M$, Ribera $M$, Monsó $E$, Lorenzo JC, Ferrándiz C. Effect of smoking on skin elastic fibres: morphometric and immunohistochemical analysis. Br J Dermatol. 2007;156(1):8591.

12. Heal CF, Buettner PG, Drobetz H. Risk factors for surgical site infection after dermatological surgery. Int J Dermatol. 2012;51(7):796-803.

13. Calzavara-Pinton P, Sala R, Arisi MC, Bussoletti C, Celleno L. Photobiology, photodermatology and sunscreens: a comprehensive overview. Part 1: damage from acute and chronic solar exposure. G Ital Dermatol Venereol. 2013;148(1):89106.

14. González-Guerra E, Pérez Suárez B, Guerra-Tapia A, Rivera Díaz R. Efectos del sol sobre la piel: bronceado y fotoprotección. Dermatología Seguimiento compartido. Tiempos Méd. 2007;639:41-6.

15. González-Guerra E, Guerra-Tapia A. Aplicación de los cosmecéuticos a la práctica dermatológica. Monogr Dermatol. 2012;25(1):63-7.

16. Valdés-Rodríguez R, Torres-Álvarez B, González-Muro J, AlmedaValdés P. The skin and the endocrine system. Gac Med Mex. 2012;148(2):162-8.

17. Henry F, Piérard-Franchimont C, Cauwenbergh G, Piérard $\mathrm{GE}$. Age-related changes in facial skin contours and rheology. J Am Geriatr Soc. 1997;45(2):220-2.

18. Brincat M, Kabalan S, Studd JW, Moniz CF, de Trafford J,
Montgomery J. A study of the decrease of skin collagen content, skin thickness, and bone mass in the postmenopausal woman. Obstet Gynecol. 1987;70(6):840-5.

19. González-Guerra E, Guerra-Tapia A. Fotoprotección. En: Castelo Branco C, Guerra-Tapia A (eds.). Envejecimiento de la piel y las mucosas. Madrid: Editorial Médica Panamericana; 2010. p. 105-12.

20. Callens A, Vaillant L, Lecomte P, Berson M, Gall Y, Lorette G. Does hormonal skin aging exist? A study of the influence of different hormone therapy regimens on the skin of postmenopausal women using non-invasive measurement techniques. Dermatology. 1996;193(4):289-94.

21. Haapasaari KM, Raudaskoski T, Kallioinen M, SuvantoLuukkonen E, Kauppila A, Läärä E, et al. Systemic therapy with estrogen or estrogen with progestin has no effect on skin collagen in postmenopausal women. Maturitas. 1997; 27(2):153-62.

22. Sauerbronn AV, Fonseca AM, Bagnoli VM, Saldiva PH, Pinotti JA. The effects of systemic hormonal replacement therapy on the skin of postmenopausal women. Int J Gynecol Obstet. 2000;68(1):35-41.

23. Owen CM, Pal L, Mumford SL, Freeman R, Isaac B, McDonald $L$, et al. Effects of hormones on skin wrinkles and rigidity vary by race/ethnicity: four-year follow-up from the ancillary skin study of the Kronos Early Estrogen Prevention Study. Fertil Steril. 2016;106(5):1170-5.e3.

24. Guerra-Tapia A, García del Cañizo C, González-Guerra E. Cosméticos y cosmecéuticos: definición, formulación y evaluación de la eficacia y seguridad. Monogr Dermatol. 2012; 25(1):5-10.

25. González-Guerra E, Guerra-Tapia A. Cuidados de la piel y las uñas del anciano. Más Dermatol. 2016;25:15-26.

26. Guerra-Tapia A, del Río R, López Barri A, González-Guerra E. Otras alternativas médicas y quirúrgicas de tratamiento del envejecimiento cutáneo. En: Castelo Branco C, Guerra-Tapia A (eds.). Envejecimiento de la piel y las mucosas. Madrid: Editorial Médica Panamericana; 2010. p. 127-32.

27. González-Guerra E, Guerra-Tapia A. ¿Qué son los hilos tensores para el descolgamiento de la piel de la cara? [Internet]. Asociación Española de Mujeres Dermatólogas (DAME). Disponible en: http://www.mujeresdermatologas.com/contenido.php?padre $=15|16| 216 \&$ IDContenido $=216$ 